\title{
Correlation of Plasma and Salivary Cortisol in Extremely Premature Infants
}

Sze M Ng ${ }^{1,2 *}$, Josephine Drury², Swathi Upradrasta ${ }^{1}$, Michael Weindling ${ }^{2}$ and Mark A Turner ${ }^{2}$

${ }^{1}$ Southport and Ormskirk Hospitals NHS Trust, Wigan Road, Ormskirk, UK

${ }^{2}$ Department of Women's and Children's Health, Institute of Translational Medicine, University of Liverpool, Liverpool, UK

\begin{abstract}
Background: In the neonatal period, cortisol concentrations are expected to increase during significant stress and are important for survival. Extreme preterm infants may develop adrenal insufficiency in the early neonatal period. Cortisol is $90 \%$ bound to cortisol binding globulins (CBG) in the circulation; therefore measurements of plasma cortisol can be compromised by conditions that alter CBG levels. Measurement of free cortisol is the best indicator of adrenal glucocorticoid secretion and can be determined in the saliva. Few studies have been reported on salivary cortisol determination in the neonatal period and particularly, extremely premature infants.
\end{abstract}

Methods: There were 65 infants (36 males). Mean gestation $25.3 \pm 1.3$ weeks. We obtained early morning plasma and salivary cortisol sampling before day 5 of postnatal age from extremely premature babies less than 28 weeks gestation. Saliva was obtained using 4 standard universal swabs by placing one swab at a time in the infant's mouth for 1-2 min. No salivary stimulants were used. Salivary cortisol was measured by competitive ELISA using a commercially available kit SLV-2930 (DRG, Germany) according to the manufacturer's instructions. Plasma cortisol was measured using DPC Immulite2000 using a solid phase 2 site chemiluminescent immunometric assay.

Results: Mean plasma cortisol levels were $400 \mathrm{nmol} / \mathrm{L} \pm 42.8 \mathrm{SEM}$, and mean salivary cortisol levels were 127.5 $\mathrm{nmol} / \mathrm{L} \pm 66.5$ SEM. Plasma cortisol was positively correlated with salivary cortisol $(r=0.41, p<0.001)$.

Conclusion: The study showed a reasonable correlation between salivary and plasma cortisol concentrations obtained early in the morning in the extremely preterm infants.

Keywords: Premature; Cortisol; Salivary

\section{Introduction}

Antenatal administration of glucocorticoids to mothers at risk of preterm labour is known to improve production of surfactant in the neonate born prematurely, thus aiding maturation of lungs. Plasma cortisol concentration is expected to increase during significant stress $[1,2]$ and recent studies in neonates by serial blood sampling have shown that cortisol is secreted in short pulses every 80 minutes [3]. These rapid pulsations are called ultradian rhythms and are caused by hypothalamic pituitary signalling. Arnold et al. [4] have shown that these secretory pulses in neonates exposed to antenatal glucocorticoids are shorter compared to neonates who have not been exposed to antenatal glucocorticoids.

Plasma cortisol concentrations are affected by intrinsic factors (for example, immaturity of the hypothalamic-pituitary-adrenal (HPA) axis, physiological decline of plasma cortisol during the immediate post-partum period, effect of antenatal steroids on HPA axis) and extrinsic factors (for example, vaginal delivery, poor condition at birth, respiratory distress, mechanical ventilation, hypoglycaemia or infection) [5]. Extremely preterm infants may develop adrenal insufficiency in the early neonatal period requiring cortisol supplementation [6,7]. In these infants, hypotension may occur which is responsive to the administration of hydrocortisone [8]. Assessment of adrenal function in extremely premature infants by the administration of ACTH for a synacthen test is difficult because sequential blood sampling is required for determination of cortisol levels.

Measurement of free cortisol is the best indicator of adrenal glucocorticoid secretion [9] because cortisol is more than $80 \%$ bound to cortisol binding globulins (CBG) in the circulation and therefore measurements of plasma cortisol can be compromised by conditions that alter CBG levels $[10,11]$. However, obtaining blood samples by venepuncture is a painful procedure, which might of itself cause high cortisol values [12] and free cortisol may be better tested by taking saliva samples [13] because salivary cortisol levels are known to accurately reflect the free fraction of cortisol in the body. Obtaining saliva is painless and non-invasive and therefore especially useful in neonates; it is unlikely to cause stress and thus salivary cortisol values are more likely to reflect the true plasma level [14].

There is good evidence from studies in older children [15] and adults that salivary cortisol concentrations correlate well with plasma cortisol concentrations at baseline/rest as well as during exercise and during dynamic function tests involving the hypothalamic-pituitary-adrenal axis [16]. These studies suggest that plasma cortisol measurement can be replaced by salivary cortisol measurement. However, few studies [17] have been reported on salivary cortisol determination in neonates and particularly, extremely premature infants.

We previously reported a novel method of collecting saliva, which is effective in obtaining a good quantity of saliva to enable reliable measurement of cortisol from the sample [14]. The aim of this present study was to evaluate the agreement between plasma and salivary cortisol levels in extremely premature infants.

*Corresponding author: Sze M Ng, Southport and Ormskirk Hospitals NHS Trust Wigan Road, Ormskirk UK, Tel: +447855084553; E-mail: may.ng@nhs.net

Received August 19, 2017; Accepted September 08, 2017; Published September 18, 2017

Citation: Ng SM, Drury J, Upradrasta S, Weindling M, Turner MA (2017) Correlation of Plasma and Salivary Cortisol in Extremely Premature Infants. J Neonatal Biol 6 : 260. doi:10.4172/2167-0897.1000260

Copyright: (c) $2017 \mathrm{Ng} \mathrm{SM}$, et al. This is an open-access article distributed unde the terms of the Creative Commons Attribution License, which permits unrestricted use, distribution, and reproduction in any medium, provided the original author and source are credited. 


\section{Methods}

This study was carried out at the Neonatal Intensive Care Unit of Liverpool Women's Hospital, UK as part of a previously published TIPIT study [18]. Extremely preterm infants under the gestational age of 28 weeks were eligible. Early morning paired saliva and blood samples were obtained from 65 extremely premature infants for plasma and salivary cortisol sampling before day 5 of postnatal age from extremely premature babies less than 28 weeks gestation. The condition of each baby at birth was measured by the CRIB [19] and Apgar scores.

There were 65 infants, of whom 36 were males. Mean gestation 25.3 \pm 1.3 weeks (range 23-27) 58 paired saliva and early morning plasma samples were obtained for measurement of cortisol level on recruitment to the study. Saliva cortisol measurements were unavailable for 7 infants due to inadequate saliva sampling.

All babies were recruited for consent. None of the infants received any postnatal corticosteroids. All mothers had received antenatal steroids prior to delivery of the infants.

Saliva was obtained using four standard universal swabs, by placing one swab at a time in the infant's mouth for one to two minutes, as previously described by $\mathrm{Ng}$ et al. [14]. No salivary stimulants were used and saliva was not obtained within half an hour of the infant having had any oral milk or formula feeds. All infants were checked for signs of blood in the mouth or saliva before each sampling. In order to prevent the samples from drying, salivary samples were centrifuged within the hour or refrigerated for a maximum period of $24 \mathrm{~h}$ prior to centrifugation. Saliva was centrifuged at $1912 \mathrm{~g}$ for $10 \mathrm{~min}$ and samples were frozen at $-80^{\circ} \mathrm{C}$ prior to analysis.

The salivary cortisol concentration was measured by competitive ELISA using a commercially available kit 'SLV-2930' (DRG, Marburg, Germany) according to the manufacturer's instructions. Plasma cortisol was measured using DPC Immulite 2000 using a solid phase 2 site chemiluminescent immunometric assay.

Standard curves were fitted by weighted regression analysis using the software Ascent (Thermo Electron Corp., Waltham, MA, USA). Commercial controls for high and low cortisol concentrations were included in every plate to determine inter- and intra-assay coefficients of variability and checked against the acceptable range published by the manufacturers.

Data was analysed using statistical software SPSS 21.0. Distributions of continuous variables were checked. P-values were calculated using a Mann Whitney U test or Spearman correlation. Statistical significance was set at $\mathrm{p}<0.05$.

The study was approved by North West Research Ethics Committee (reference number 07/MRE08/37) and by the Medicines for Human Regulatory Agency (MHRA). The parents of each potentially eligible baby were informed of the study's objectives and overall requirements after birth when the baby had achieved respiratory and haemodynamic stability. The Investigator explained the study fully to the patient's parent(s)/guardian(s) using the Patient Information Leaflet. The parent/guardian was then given at least $12 \mathrm{~h}$ to consider the study. If a parent/guardian was willing for the patient to participate in the study written informed consent was obtained.

\section{Results}

Mean plasma cortisol levels were $400 \mathrm{nmol} / \mathrm{L} \pm 42.8 \mathrm{SEM}$ and mean salivary cortisol levels were $127.5 \mathrm{nmol} / \mathrm{L} \pm 66.5 \mathrm{SEM} .100 \mu \mathrm{L}$ of saliva was obtained at each sampling by trained staff in $85 \%$ of the cases. Volume of saliva obtained ranged from $25-250 \mu \mathrm{L}$ in all babies.

Median plasma and salivary cortisol levels with inter-quartile range (IQR) are shown in Table 1 . There was a statistically significant correlation between plasma cortisol and salivary cortisol $(\mathrm{r}=0.413$, $\mathrm{p}=0.001$ ). This is shown in Figure 1. A Bland Altman plot for difference between plasma cortisol and salivary cortisol is shown in Figure 2 which showed good agreement between plasma and salivary cortisol levels particularly at lower cortisol levels, whilst there seems to be a systematic trend for plasma cortisol measurements to be higher than those for salivary cortisol. There was no difference in plasma or salivary cortisol secretion between boys and girls $(\mathrm{p}=0.8, \mathrm{p}=0.40$, respectively). Salivary cortisol levels were significantly lower in infants born by caesarean section $(\mathrm{P}=0.028)$ and a similar trend was seen in plasma cortisol levels $(\mathrm{P}=0.058)$. Median CRIB score was 5 with a range of 1-14. There was no significant association between CRIB

\begin{tabular}{|l|c|}
\hline Variable & Median (IQR) \\
\hline Gestation (weeks) & $25(23-27)$ \\
\hline Weight (g) & $780(690-905)$ \\
\hline Plasma cortisol (nmol/L) & $270(165-494)$ \\
\hline Salivary cortisol (nmol/L) & $76.7(31.0-192.6)$ \\
\hline
\end{tabular}

IQR: Interquartile Range

Table 1: Demographics.

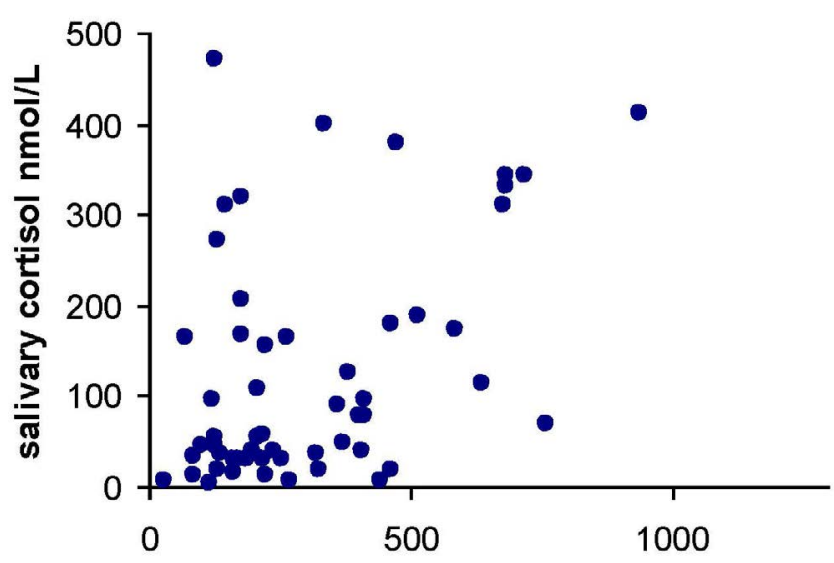

Plasma cortisol nmol/L

$r=0.41, p<0.001$

Figure 1: Correlation between plasma and salivary cortisol.

Bland Altman plot

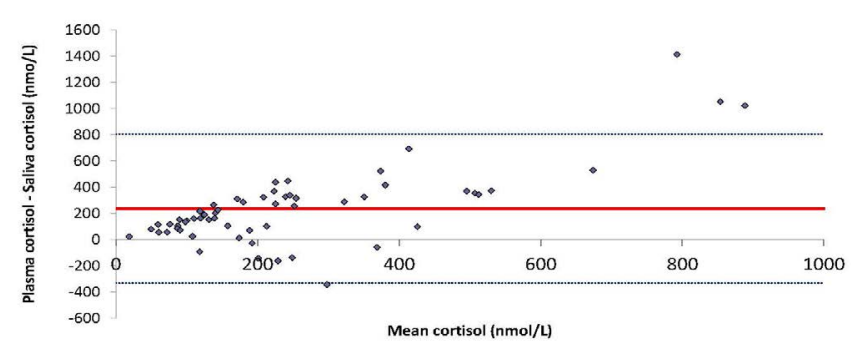

Figure 2: Bland Altman plot for the difference between plasma cortisol and salivary cortisol and mean cortisol. 
scores and plasma or salivary cortisol levels of the infants $(\mathrm{p}=0.089$, $\mathrm{p}=0.21$, respectively).

There was no association between gestation and either plasma or salivary cortisol levels ( $\mathrm{r}=-0.207, \mathrm{p}=0.097 ; \mathrm{r}=-0.198, \mathrm{P}=0.137$, respectively). There was no correlation between birth weight and plasma or salivary cortisol levels of the infants $(\mathrm{p}=0.19, \mathrm{P}=0.22$. respectively). There was no evidence of instability of cortisol on the cotton swabs during storage in the refrigerator for periods up to $24 \mathrm{~h}$ [14].

\section{Discussion}

The current practice in neonatal and paediatric units in the United Kingdom to evaluate the adrenal function is by measuring plasma cortisol levels of neonates, either as a single early morning sample or in response to ACTH as part of synacthen test. However, this is frequently restricted due to the necessity of sequential blood sampling for determination of cortisol levels following administration of ACTH during synacthen test. Measurement of salivary cortisol is considered useful due to the ease of obtaining samples. The procedure of obtaining saliva is painless and free from stress to the infants [18]. A few studies have reported on the relationship between plasma and salivary cortisol in term [20-22] infants.

This present study found a reasonable agreement between plasma and salivary cortisol concentrations in extremely preterm infants at lower cortisol levels and there seems to be a systematic trend for plasma cortisol measurements to be higher than those for salivary cortisol.

Matsukura et al. reported good correlation between plasma and salivary cortisol levels in preterm infants (mean gestational age at birth 26.7 weeks) during Corticotropin Releasing Hormone (CRH) stimulation test [20]. Calixto et al. reported a good correlation between salivary and plasma cortisol levels in preterm infants during an ACTH stimulation test [17] in a study population of mean gestational age of 30.6 weeks at birth. In that study, plasma and salivary cortisol levels were correlated at baseline $(\mathrm{r}=0.67$ and $\mathrm{p}<0.0001)$ as well as following stimulation with ACTH $(r=0.40, \mathrm{p}=0.005)$.

Although one recent study by Maas et al. [21] reported unsatisfactory agreement between plasma and salivary cortisol, this was particularly for calculation of a predicted plasma cortisol value from the obtained salivary cortisol value. No correlation values were reported for comparison of plasma and salivary cortisol concentrations in their study. They also reported low sensitivity and specificity for prediction of adrenal insufficiency (0.66 and 0.62 , respectively).

One study by Chou et al. [22] reported good correlation between cord blood cortisol and salivary cortisol levels in preterm neonates $(\mathrm{r}=0.48$ and $\mathrm{p}=0.0003)$. They obtained cord blood from umbilical cord vessels or placental vessels immediately after clamping the umbilical cord.

Results of studies on term neonates are also in agreement with our findings. Francis et al. demonstrated good correlation between salivary and plasma cortisol levels in full term infants $(r=0.83)$ [23]. Interestingly, they demonstrated no difference in salivary cortisol levels with environmental factors such as light, darkness or time of the day. Kurihara et al. also reported good correlation of plasma and salivary cortisol levels in term neonates on day 5 of life $(r=0.718, p<0.0001)$ [12].

New-born infants produce minimal quantities of saliva and several previous studies used salivary stimulants to enhance the production of saliva. Citric acid was used as a salivary stimulant by Francis et al. [23] and Calixto et al. [17]. Unsweetened lemon crystals were used by
Kurihara et al. [12] for stimulation of saliva. However, these stimulants may affect the concentration of cortisol in saliva [24]. Francis et al. [23] used suction of oral cavity to obtain saliva. However, this can cause damage to the delicate oral mucosa of the preterm neonates. Maas et al. [21] and Matsukura et al. [20] used no salivary stimulants. However, both studies report a high rate of infants that could not be studied due to inadequate quantity of saliva obtained. Matsukura et al. reported that $50 \%$ of preterm infants (9/17) were excluded from the study; quantity of saliva obtained was not reported. Maas et al. reported the exclusion of $49 \%$ of preterm infants (28/58); they obtained mean volume of $53 \mu \mathrm{L}$ of saliva (range 10-80 $\mu \mathrm{L}$ ). Chou et al. [22] report the use of Salivette (Sarstedt) with no salivary stimulants, however, they did not report on the quantity of saliva obtained by this method. No salivary stimulants were used in our study and we succeeded in obtaining mean volume of 100 microliters of saliva at each sampling (Range 25-200 $\mu \mathrm{L}$ ).

Greaves et al. [25] described significantly higher levels of plasma cortisol levels in "younger" preterm infants (born at 23-26 weeks gestation) compared to "older" preterm infants (born at 27-29 weeks gestation). They reported a mean difference in cortisol levels of -1104 $\mathrm{nmol} / \mathrm{L}$ per week of gestation ( $\mathrm{p}<0.001,95 \%$ confidence intervals -1619 to $-589 \mathrm{nmol} / \mathrm{L}$ ) by univariate analysis. In contrast to previous studies, we found no correlation between plasma or salivary cortisol levels and gestation at birth of preterm infants. This is likely to be due to the restricted gestational age range in this study.

Gender dimorphism has been linked with differences in circulating peptides and steroid hormones [26]. Studies have shown no difference in basal salivary cortisol in children aged 1-15 years in the mornings and evenings [27]. In our study, we found no difference between salivary cortisol secretions between genders.

One of the limitations of this study is the small sample size. In addition, although we have previously reported a novel and effective method of obtaining saliva from extremely preterm infants [14], we were still not able to analyse 7 patient samples due to insufficient quantity of collected saliva.

There is a need to standardise the method of collection of saliva as several studies have reported the use of salivary stimulants, which may affect the cortisol levels, as detailed above. There is also a need to establish reference ranges for normal values of salivary cortisol levels, in order to use salivary cortisol to assess adrenal function. There is a need for different range of normal values for cortisol levels at different gestational ages, as cortisol levels have been found to be higher at term compared to two weeks age. These issues will require further research in a larger population group, which would be best performed as a multi-centre study.

\section{Conclusion}

In conclusion, our study showed a reasonable agreement between salivary and plasma cortisol concentrations in the extremely preterm infants and this methodology should be considered in assessment of cortisol reserve in the extremely premature infant.

\section{References}

1. Widmer IE, Puder JJ, Konig C, Pargger H, Zerkowski HR, et al. (2005) Cortisol response in relation to the severity of stress and illness. J Clin Endocrinol Metab 90: 4579-4586.

2. Watterberg $\mathrm{KL}$ (2004) Adrenocortical function and dysfunction in the fetus and neonate. Semin Neonatol 9: 13-21.

3. Metzger DL, Wright NM, Vendhuis JD, Rogol AD, Kerrigan JR (1993) Characterization of pulsatile secretion and clearance of plasma cortisol in premature and term neonates using deconvolution analysis. J Clin Endocrinol Metab 77: 458-463. 
Citation: Ng SM, Drury J, Upradrasta S, Weindling M, Turner MA (2017) Correlation of Plasma and Salivary Cortisol in Extremely Premature Infants. J Neonatal Biol 6: 260. doi:10.4172/2167-0897.1000260

4. Arnold JD, Bonacruz G, Leslie GI, Veldhuis JD, Milmlow D, et al. (1998) Antenatal glucocorticoids modulate the amplitude of pulsatile cortisol secretion in premature neonates. Pediatr Res 44: 876-881

5. Ng PC (2008) Is there a "normal" range of serum cortisol concentration for preterm infants? Paediatrics 122: 873-875.

6. Ng PC, Lee CH, Lam CWK, Ma KC, Fok TF, et al. (2004) Transient adrenocortical insufficiency of prematurity and systemic hypotension in very low birthweight infants. Arch Dis Child Fetal Neonatal Ed 89: F119-F126.

7. Heckmann M, Hartmann MF, Kampschulte B, Gack H, Bodeker RH, et al. (2005) Cortisol production rates in preterm infants in relation to growth and illness: A non-invasive prospective study using gas chromatography - mass spectrometry. J Clin Endocrinol Metab 90: 5737-5742.

8. Helbock HJ, Insoft RM (1993) F.A. C: Glucocorticoid responsive hypotension in extremely low birth weight newborns. Pediatrics 92: 715-717.

9. Le Roux CW, Chapman GA, Kong WM, Dhillo WS, Jones J, et al. (2003) Free cortisol index is better than serum total cortisol in determining hypothalamicpituitary-adrenal status in patients undergoing surgery. J Clin Endocrinol Metab 88: 2045-2048.

10. Brien TG (1981) Human corticosteroid binding globulin. Clin Endocrinol 14: 193-212.

11. Dhillo WS, Kong WM, Le Roux CW, Alaghband-Zadeh J, Jones J, et al (2002) Cortisol-binding globulin is important in the interpretation of dynamic tests of the hypothalamic-pituitary-adrenal axis. Eur J Endocrinol 146: 231-235.

12. Kurihara H, Chiba H, Shimizu Y, Yanaihara T, Takeda M, et al. (1996) Behavioral and adrenocortical responses to stress in neonates and the stabilizing effects of maternal heartbeat on them. Early Hum Dev 46: 117-127.

13. Tornhage CJ (2009) Salivary cortisol for assessment of hypothalamic-pituitaryadrenal axis function. Neuroimmunomodulation 16: 284-289.

14. Ng SM, Drury JA, Turner MA, Didi M, Victor S, et al. (2013) A novel method of collection of saliva for estimation of steroid levels in extremely premature infants. Acta Paediatr 102: 356-359.

15. Kiess W, Meidert A, Dressendorfer RA, Schriever K, Kessler U, et al. (1995) Salivary cortisol levels throughout childhood and adolescence: Relation with age, pubertal stage and weight. Pediatr Res 37: 502-506

16. Gozansky WS, Lynn JS, Laudenslager ML, Kohrt WM (2005) Salivary cortisol determined by enzyme immunoassay is preferable to serum total cortisol for assessment of dynamic hypothalamic-pituitary-adrenal axis activity. Clin Endocrinol 63: 336-341.

17. Calixto C, Martinez FE, Jorge SM, Moreira AC, Martinelli Jr CE (2002) Correlation between plasma and salivary cortisol levels in preterm infants. $J$ Pediatr 140: 116-118.

18. Ng SM, Turner MA, Gamble C, Didi M, Victor S, et al. (2013) An explanatory randomised placebo controlled trial of levothyroxine supplementation for babies born $<28$ weeks' gestation: Results of the TIPIT trial. Trials 14: 211.

19. The International Neonatal Network (1993) The CRIB (clinical risk index for babies) score: A tool for assessing initial neonatal risk and comparing performance of neonatal intensive care units. Lancet 342: 193-198.

20. Matsukura T, Kawai M, Marumo C, Iwanaga K, Yoshida K, et al. (2012) Diagnostic value of salivary cortisol in the $\mathrm{CRH}$ stimulation test in premature infants. J Clin Endocrinol Metab 97: 890-896.

21. Maas C, Ringwald C, Weber K, Engel C, Poets CF, et al.( 2014) Relationship of salivary and plasma cortisol levels in preterm infants: Results of a prospective observational study and systematic review of the literature. Neonatology 105 312-318.

22. Chou IC, Lien HC, Lin HC, Fu JJC, Kao CH, et al. (2010) The relationship of salivary and cord blood cortisol in preterm infants. J Pediatr Endocrinol Metab 23: $1-5$.

23. Francis S, Walker R, Riad-Fahmy D, Hughes D, Murphy J, et al. (1987) Assessment of adrenocortical activity in term newborn infants using salivary cortisol determinations. J Pediatr 111: 129-133.

24. Schwartz EB, Granger DA, Susman EJ, Gunnar MR, Laird B (1998) Assessing salivary cortisol in studies of child development. Child Dev 69:1503-1513.

25. Greaves RF, Zacharin MR, Donath SM, Inder TE, Doyle LW (2014) Establishment of hormone reference intervals for infants born $<30$ weeks gestation. Clin Biochem 47: 101-108.

26. Kajantie E, Phillips DI (2006) The effects of sex and hormonal status on the physiological response to acute psychosocial stress. Psychoneuroendocrinology 31: 151-178.

27. Groschl M, Rauh M, Dorr HG (2003) Circadian rhythm of salivary cortisol, 17 alpha-hydroxy progesterone and progesterone in healthy children. Clin Chem 49: $1688-1691$. 